\title{
ANÁLISE TÉRMICA APLICADA AO ESTUDO DE MATERIAIS ENERGÉTICOS
}

\author{
Jony Andrade, Koshun Iha*, Jose Atílio Fritz Fidel Rocco e Eduardo Marcelo Bezerra \\ Instituto Tecnológico de Aeronáutica, Pr. Ma. Eduardo Gomes, 50, 12228-900 São José dos Campos- SP, Brasil \\ Maria Encarnación Vázquez Suárez-Iha \\ Instituto de Química, Universidade de São Paulo, Av. Prof. Lineu Prestes, 748, São Paulo-SP, Faculdade de Ciências Biológicas, \\ Exatas e Experimentais, Universidade Presbiteriana Mackenzie, Rua da Consolação, 930, São Paulo - SP, Brasil \\ Glaci Ferreira Martins Pinheiro \\ Divisão de Química, Instituto de Aeronáutica e Espaço, Pr. Ma. Eduardo Gomes, 50, 12228-904 São José dos Campos - SP, Brasil
}

Recebido em 15/3/06; aceito em 18/10/06; publicado na web em 27/4/07

\begin{abstract}
THERMAL ANALYSIS APPLIED TO THE STUDY OF ENERGETIC MATERIALS. This paper shows different aspects related to the application of different thermal analysis techniques in the study of energetic materials. The criteria used to choose the best technique and an exact approach to adjust the experimental data with a proper model are here discussed. The paper shows how to use the different thermal analysis results to help develop new compounds, to study the stability of some energetic materials and their compatibility, and the conditions necessary for a secure storing environment.
\end{abstract}

Keywords: thermal analysis; energetic material; propellants.

\section{INTRODUÇÃO}

Materiais energéticos são utilizados tanto na área civil como militar; compostos como nitroglicerina, pólvora negra e nitrocelulose estão entre os mais utilizados ao longo das últimas décadas. Entre as propriedades características destes compostos e/ou formulações, pode-se destacar a capacidade de liberarem grande quantidade de energia em curto espaço de tempo, quando expostos a estímulo térmico, elétrico ou em decorrência de um impacto. Em virtude destas características, inúmeros programas de pesquisa vêm sendo desenvolvidos, tendo como enfoque o aumento da performance destes materiais em tais propriedades ${ }^{1}$.

As diversas técnicas termoanalíticas ${ }^{2}$ utilizadas isoladamente ou em conjunto com outras técnicas, tais como espectroscopia no Infravermelho (IR), espectroscopia de massas (MS), cromatografia gasosa (CG) ou cromatografia líquida de alta eficiência (CLAE) vêm demonstrando serem ferramentas importantes no estudo dos materiais energéticos. A análise térmica diferencial (DTA), a calorimetria exploratória diferencial (DSC) e a termogravimetria (TG) podem ser utilizadas, por ex., na compreensão dos mecanismos físico-químicos relativos a processos de decomposição térmica ${ }^{3}$ ou no estudo e desenvolvimento de novos compostos ${ }^{4}$, entre outros.

Neste trabalho, é apresentada uma discussão sobre a aplicação das técnicas termoanalíticas ao estudo de compostos altamente energéticos. São discutidas diferentes condições necessárias ao desenvolvimento de estudos experimentais e apresentados diversos exemplos de aplicações da termoanálise a materiais energéticos.

\section{ESTUDO CINÉTICO DO COMPORTAMENTO TÉRMICO DE MATERIAIS ENERGÉTICOS VIA ANÁLISE TÉRMICA}

Nas últimas décadas, as técnicas termoanalíticas têm sido amplamente utilizadas para estudo de materiais energéticos ${ }^{5}$. São empregadas principalmente para obtenção de dados referentes à cinética de degradação térmica de diferentes formulações ${ }^{6}$. Os

*e-mail: koshun@ita.br parâmetros desejados são obtidos a partir das curvas TG ou DSC, ou ainda, por microcalorimetria. A busca de tais informações tem como objetivo o aperfeiçoamento e o desenvolvimento de novos materiais. Além disso, os diversos estudos realizados permitem encontrar as condições mais adequadas para processos de estocagem, para o desenvolvimento de novos estabilizantes que retardem processos de degradação oxidativa e/ou térmica, além da obtenção de dados termodinâmicos ${ }^{7}$.

Entre os principais estudos efetuados por meio da DSC, destacam-se a obtenção do grau de cura de polímeros, percentual de cristalização, obtenção de temperatura de transição vítrea e a determinação do calor específico de uma amostra, que é calculado a partir das medidas da potência requerida para manter uma elevação de temperatura em uma dada razão de aquecimento, quando comparada com um material de referência ${ }^{8}$. $\mathrm{O}$ estudo cinético da decomposição térmica de materiais energéticos é realizado, de modo geral, mediante a adoção de um dos vários modelos cinéticos encontrados na literatura ${ }^{9}$ a aplicação de uma determinada técnica termoanalítica ${ }^{10}$. Utilizando resultados obtidos a partir de curvas DSC, Kishore ${ }^{11}$ apresentou um modelo relativo à cinética de decomposição térmica dos sólidos explosivos ciclotetrametilenotetranitroamina (HMX) e ciclotetrametileno-trinitroamina (RDX). O modelo desenvolvido teve como base a suposição de que os mecanismos cinéticos envolvidos na reação de decomposição térmica, para um determinado composto, são independentes da extensão de conversão da amostra e, portanto, tem-se uma ordem de reação constante. Assim, a variação da massa da amostra, decomposta isotermicamente, em função do tempo $(\mathrm{dm} / \mathrm{dt})$ pode ser calculada pela razão entre a área da curva obtida em um determinado tempo e a área total.

Partindo-se da expressão geral que representa a cinética de uma reação, Equação 1,

$\frac{d a}{d t}=k(1)-a^{n}$

onde $\alpha$ é a fração de conversão da amostra e "n" a ordem de rea- 
ção, e utilizando-se a Equação de Arrhenius,

$k=A e^{-E a / R T}$

onde "k" é a constante de velocidade, "A" o fator freqüência, "Ea" a energia de ativação, "R" a constante universal dos gases e "T" a temperatura absoluta, obtém-se a seguinte expressão:

$d a / d t=A e^{-E a / R T}(1-a)^{n}$

Como a variação da massa está relacionada à área da curva obtida, e consequientemente a área da curva à variação de entalpia $(\Delta \mathrm{H})$ do processo de decomposição, tem-se a Equação,

$\mathrm{Z} / \Delta \mathrm{H}=A e^{-E a / R T}(1-a)^{n}$

onde $\mathrm{Z}$ representa a taxa de conversão instantânea. Considerandose $\alpha$ e n constantes, o termo $A(1-\alpha)^{\mathrm{n}}$ da Equação 4 pode ser considerado constante e representado por $b$ :

$b=A(1)-a^{n}$

A Equação 4 pode, então, ser reescrita como:

$Z / \Delta H=b e^{-E a / R T}$

e linearizada, obtendo-se:

$\log (Z / \Delta \mathrm{H})=-\frac{E_{a}}{R T}+\log b$

A energia de ativação (Ea) pode ser calculada, portanto, a partir do coeficiente angular do gráfico $\log \mathrm{Z} / \Delta \mathrm{H} v s$. $1 / \mathrm{T}$.

$\mathrm{O}$ modelo apresentado por Kishore ${ }^{11}$ tem como base os valores de entalpia obtidos a partir das curvas geradas com uso da técnica DSC. Outros modelos, entretanto, têm como base os dados obtidos a partir das curvas termogravimétricas (TG) e, de modo geral, os fundamentos teóricos dos métodos são os mesmos que os adotados para DSC, diferenciando-se apenas no monitoramento da variação de entalpia (DSC) ou da variação de massa (TG), ambos em função de temperatura ou tempo.

Quando condições experimentais semelhantes são utilizadas para o estudo cinético de um composto, a aplicação de diferentes modelos cinéticos leva geralmente a variações nos dados coletados, conforme avaliação feita por Singh et al. ${ }^{12}$. A energia de ativação para a decomposição térmica de RDX foi obtida pelo método de ajuste, método de Kishore ${ }^{11}$ e também pelo método isoconversional, resultando em valores de 157 e $170 \mathrm{~kJ} / \mathrm{mol}$, respectivamente, e para o isoconversional valores entre 200 a $150 \mathrm{~kJ} / \mathrm{mol}$. Os valores para a energia de ativação do RDX, obtidos pelo método isoconversional, são apresentados dentro de um intervalo, uma vez que o cálculo é realizado para diferentes frações de conversão da amostra. Segundo Long e Vyazovkin ${ }^{13}$, estudos cinéticos por meio do método isoconversional oferecem condições mais apropriadas para análise das diferentes etapas ocorridas em um processo de termólise, pois descreve sua variação ao longo do processo de decomposição, evidenciando a existência de diferentes processos e mecanismos. Este comportamento foi verificado por Singh et al..$^{12}$ no estudo cinético da degradação do RDX, principalmente na fase líquida e em três etapas de decomposição: vaporização, decomposição da fase líquida e decomposição da fase gasosa, com diferentes valores da energia de ativação para cada evento.

Nos estudos da cinética de decomposição térmica de materiais energéticos, os parâmetros cinéticos relativos ao processo, como a energia de ativação (Ea) e o fator Freqüência (A), além da obtenção da variação de entalpia do processo $(\Delta \mathrm{H})$, são obtidos para certa região de temperatura. O comportamento relativo a outras faixas de temperatura é geralmente obtido por extrapolação do modelo de decomposição. Esta extrapolação é aplicada também à simulação da decomposição térmica do material energético no decorrer do tempo de armazenamento.

Deve-se salientar, no entanto, que uma análise crítica dos dados obtidos a partir de extrapolações deve ser realizada, pois, quando uma das variáveis empregadas é a temperatura, essa extrapolação pode não representar os mecanismos de reação que realmente ocorrem. Outro aspecto importante a ser avaliado, apesar do grande avanço tecnológico obtido nos últimos anos, com a disponibilidade de aparelhos termoanalíticos com maior precisão e exatidão, refere-se à sensibilidade do aparelho; a técnica adotada deve oferecer dados com alta confiabilidade.

Estas considerações foram feitas por Bunyam et al. ${ }^{14}$ no estudo cinético de decomposição térmica de uma amostra de RDX em propilenoglicol. A variação de energia detectada com o uso de um microcalorímetro, quando a amostra foi submetida à decomposição isotérmica nas temperaturas de $60,70,80$ e $90{ }^{\circ} \mathrm{C}$, não era concordante com dados previamente calculados por extrapolação a partir de curvas DSC, obtidas nas temperaturas de $200,205,210$ e $215^{\circ} \mathrm{C}$. Os valores detectados pelo microcalorímetro para as energias liberadas pela amostra foram superiores aos estimados por DSC. As diferenças observadas poderiam estar relacionadas a interferências produzidas pelo próprio aparelho, ou ainda a possíveis reações secundárias ocorridas durante o processo de termólise. Os autores ${ }^{14}$ concluíram, portanto, que o estudo da decomposição térmica utilizando o microcalorímetro $\left(60\right.$ a $90{ }^{\circ} \mathrm{C}$ ), como ferramenta para determinar o tempo de vida útil do PBX, baseado em RDX e propilenoglicol, é difícil de ser realizado, em virtude das baixas energias envolvidas, consumindo muito tempo e tornando-se, assim, dispendioso.

Outro fator importante a ser observado em estudos sobre a cinética de decomposição térmica de materiais energético é o tipo de porta-amostra a utilizar. A geração de um grande número de espécies químicas gasosas durante a decomposição de materiais energéticos, seguida ainda de um processo altamente exotérmico ${ }^{15}$, leva sempre a um aumento da pressão interna no suporte em que a amostra é colocada, levando a possíveis alterações na curva gerada e até, em casos mais extremos, à abertura do porta-amostra, oferecendo assim sérios riscos ao aparelho. Em decorrência disto, observa-se na literatura algumas indicações quanto à condição de uso do porta-amostra; se aberto, fechado ou fechado com um furo na tampa. Singh et al. $^{12}$ apresentaram um estudo sobre a decomposição térmica do RDX, utilizando os três tipos de porta-amostra citados. Os resultados obtidos para a fração de decomposição, as temperaturas e a variação de entalpia não evidenciam diferenças em função dos diferentes porta-amostras utilizados. Kimura e Kubota ${ }^{16}$ relataram um trabalho sobre a decomposição térmica de uma amostra de HMX utilizando curvas DSC e TG, obtidas utilizando um porta-amostra aberto e um fechado contendo um furo na tampa. Neste trabalho, da mesma maneira, não foram obtidas diferenças significativas entre os resultados.

Ainda em relação à condição de uso do porta-amostra, pode-se destacar que o porta-amostra aberto oferece a vantagem da possibilidade de coleta dos produtos gasosos gerados durante a decomposição e a respectiva identificação dos produtos gasosos por meio de técnicas como a espectroscopia de infravermelho (IR) e espectroscopia de massas $(\mathrm{MS})^{17}$ que podem ser acopladas às técnicas termoanalíticas.

A coleta individual dos gases de combustão consiste basicamente da utilização de um tubo de vidro, próprio para acondicio- 
namento de amostras energéticas, onde a amostra é decomposta termicamente e os gases formados ficam armazenados, sendo identificados por análise espectroscópica ${ }^{18}$. Entretanto, este método de coleta dos produtos de combustão, apesar de apresentar boa reprodutibilidade, não propicia a exatidão necessária ao ajuste de modelos que representem os mecanismos relativos à termólise do composto em estudo. Isto se deve ao fato de a razão de aquecimento adotada, quando o material é decomposto termicamente dentro do tubo de vidro, ser diferente da utilizada na termoanálise e esta diferença tem influência significativa nos mecanismos da reação de combustão. Este inconveniente pode, no entanto, ser minimizado com a utilização de técnicas acopladas, o que oferece a vantagem da identificação dos gases de combustão simultaneamente à obtenção da curva termoanalítica garantindo, assim, que os produtos gasosos identificados representem verdadeiramente os mecanismos de rupturas das ligações do composto durante o processo de termólise ${ }^{19}$

\section{ESTUDO DE MATERIAIS ENERGÉTICOS VIA ANÁLISE TÉRMICA}

Parâmetros como mecanismos de decomposição, temperatura de decomposição, variação de entalpia de combustão, grau de conversão da amostra, capacidade calorífica, compatibilidade química e sensibilidade à fricção e atrito são informações essenciais para estudos comparativos entre diferentes materiais energéticos ${ }^{20}$. Podese destacar, de modo geral, que entre os parâmetros citados a temperatura de decomposição de um explosivo é um dos principais pontos a ser analisado em um estudo de estabilidade ${ }^{21}$, como pode ser observado no trabalho realizado por Talawar et al. ${ }^{22}$. Neste trabalho, as temperaturas de decomposição térmica do mercúrio-5-nitrotetrazolato (MNT) e do explosivo primário azoteto de chumbo $\mathrm{Pb}\left(\mathrm{N}_{3}\right)_{2}$, obtidas a partir de curvas DTA nas razões de aquecimento de 5 e $10^{\circ} \mathrm{C} / \mathrm{min}$, foram utilizadas como parâmetro para comparar as estabilidades destes explosivos. Os autores concluíram que o azoteto de chumbo, com as temperaturas de decomposição de 327 e $345{ }^{\circ} \mathrm{C}$, apresentava uma estabilidade térmica maior que o MNT, caracterizado pelas temperaturas de decomposição 228 e $230{ }^{\circ} \mathrm{C}$.

A temperatura de decomposição térmica não deve, entretanto, ser considerada um parâmetro determinante em estudos de materiais explosivos, uma vez que, dados relativos aos testes de compatibilidade química e estabilidade à fricção fornecem uma visão mais ampla quanto as características do material ${ }^{23}$. Uma confirmação sobre a compatibilidade química entre compostos participantes de uma formulação é obtida quando a temperatura de decomposição térmica da formulação resultante é menor em relação aos compostos originais. A obtenção de um composto com alta sensibilidade a estímulos físicos ${ }^{24}$, entre outros, evidencia uma incompatibilidade entre compostos.

Portanto, para a obtenção de uma análise mais conclusiva quanto ao potencial de um composto, é necessário um conjunto de informações que venham a oferecer uma base de dados para comparação com outros compostos, como pode ser observado no trabalho de Felix et al. ${ }^{25}$. Os autores relataram um estudo sobre o explosivo 2-oxo-1,3,5-triazocicloexano (K-6), onde obtiveram dados relativos à densidade $\left(\rho=1,93 \mathrm{~g} \mathrm{~cm}^{-3}\right)$, ao calor de formação $(-41,9 \pm 4,2$ $\mathrm{kJ} / \mathrm{mol}$ ), à variação de entalpia e aos produtos gasosos gerados durante a decomposição, entre outros. Este conjunto de informações reunidas permitiu considerar a possível utilização do K-6 em formulações de propelentes sólidos para propulsores de mísseis ${ }^{26}$.

Para uma análise mais detalhada do K-6, curvas TG foram obtidas sob atmosfera estática de ar em condições não isotérmicas e uma razão de aquecimento de $10{ }^{\circ} \mathrm{C} / \mathrm{min}$. Da decomposição térmica resultou uma perda de massa de aproximadamente $89 \%$, em uma única etapa, em temperatura inicial de $184{ }^{\circ} \mathrm{C}$ e final de 201 ${ }^{\circ} \mathrm{C}$. Estas informações associadas às anteriormente citadas permitiram, por ex., evidenciar que o composto K-6 é menos estável termicamente quando comparado ao RDX, ou seja, tende a se decompor termicamente em temperaturas inferiores, indicando que o K-6 está sujeito a perder as suas propriedades físico-químicas mais facilmente, além de ser mais suscetível a um processo de autoignição. Os autore ${ }^{25}$ relataram que os dados obtidos para a decomposição térmica do K-6 são concordantes com Otsmark et al. ${ }^{27}$.

Como destacado por Felix et al. ${ }^{25}$, as curvas TG oferecem basicamente o percentual de conversão da amostra e o número de etapas ocorridas, sendo que uma alta fração de conversão em um número mínimo de etapas indica boas características para um explosivo ${ }^{28}$. Outro ponto a salientar no estudo de explosivos é a obtenção da energia de ativação a partir de curvas DSC ou TG. Este importante parâmetro fornece uma avaliação direta da energia necessária para o início da decomposição do composto, demonstrando assim a estabilidade do composto. Este parâmetro de avaliação foi o adotado no trabalho de Ostmark et al..$^{29}$ que avaliou a estabilidade do explosivo FOX-12 a partir da energia de ativação ( $\mathrm{E}_{\mathrm{a}}$ ) obtida pelas curvas DSC e TG. O resultado encontrado igual a $277 \mathrm{~kJ} / \mathrm{mol}$, quando comparado ao $\mathrm{RDX}^{30}$ que apresenta um valor de $201 \mathrm{~kJ} / \mathrm{mol}$, permitiu aos autores concluir que o FOX-12 é mais estável termicamente.

O desempenho de um material energético está também relacionado à identidade e à quantidade de produtos gasosos gerados durante a decomposição térmica. Assim, em estudos termoanalíticos a caracterização do composto por IR e RAMAN em conjunto com a análise elementar dos constituintes vem adicionar dados para avaliação dos gases a serem gerados, sendo que, em alguns casos podem definir o desempenho do material em termos de suas propriedades energéticas ${ }^{31}$.

Compostos de elevada massa molar e baixo volume podem apresentar uma estrutura mais compacta, com alta densidade e maior estabilidade, conferindo assim a capacidade de produzir uma grande quantidade de mols de produtos gasosos, quando submetidos ao processo de combustão. Conciliando o grande número de produtos gasosos formados durante a combustão com a alta energia produzida durante este processo, que se deve principalmente à presença de átomos de nitrogênio, têm-se então as duas principais propriedades que caracterizam um explosivo.

Uma visão mais detalhada sobre os mais recentes explosivos desenvolvidos e sobre os mais utilizados é relatada por Sikder et $a l .^{32}$, com uma revisão sobre compostos com boas perspectivas para utilização em formulações de propelentes sólidos e explosivos, onde as técnicas termoanalíticas foram utilizadas para obtenção das suas propriedades.

\section{TEMPERATURA CRÍTICA E COMPATIBILIDADE}

Ao longo da história são relatados inúmeros incidentes com explosivos, em virtude de problemas como estocagem inadequada e incompatibilidade com outros compostos ${ }^{33}$. Para a prevenção de ocorrências deste tipo, estudos relativos à temperatura crítica de um material energético são muito importantes, pois fornecem a temperatura na qual um determinado material poderá sofrer auto-ignição ${ }^{34}$.

A determinação dos valores da temperatura crítica permite o planejamento das condições mais adequadas de armazenamento para os materiais energéticos ${ }^{35}$. Por ex., Tonglay et al. ${ }^{36}$ realizaram um estudo sobre a determinação da temperatura crítica para o RDX, a partir de dados obtidos em curva DSC nas razões de aquecimento de $1,2,5,10$ e $20 \mathrm{~K} / \mathrm{min}$ e apresentaram uma fórmula para o cálculo da temperatura crítica de explosão térmica de materiais energéticos. Esta Equação é derivada a partir da teoria de explosão 
térmica de Semenov ${ }^{37}$ e da Equação cinética no modo não isotérmico:

$\frac{d \alpha}{d t}=a f(\alpha) e^{\frac{-E a}{R T}}$

onde " $\alpha$ " é a fração de conversão da amostra; "t" o tempo; "A" o fator frequiência; " $E_{a}$ " a energia de ativação; "R" a constante universal dos gases e "T" que é a temperatura onde é observado o início da deflexão da curva térmica, obtida a partir da linha base estabelecida, que é também conhecida como temperatura onset $\left(\mathrm{T}_{\text {onset }}\right)$.

A diferenciação a partir da teoria de $\operatorname{Semenov}^{37}$, em conjunto com a Equação cinética, irá resultar na expressão,

$E_{a}\left(T_{b}-T_{\text {onset }}\right)-R T_{b}^{2}=0$

onde $\mathrm{E}_{\mathrm{a}}$ é a energia de ativação obtida por meio da análise no modo não isotérmico das curvas DSC, sendo calculada a partir do coeficiente angular, plotando-se a variação da temperatura de pico $(1 / \mathrm{T})$ em função da variação da razão de aquecimento; " $\mathrm{T}$ onset " a temperatura onset; $\mathrm{e}$ " $\mathrm{T}_{\mathrm{b}}$ " a temperatura crítica. Tonglay et al. ${ }^{36}$ obtiveram a temperatura de ignição do RDX como sendo $482 \mathrm{~K}$ e concluíram pela concordância com o valor estabelecido na literatura, que é de $488 \mathrm{~K}$.

A avaliação dos riscos oferecidos por compostos energéticos não se restringe apenas à obtenção da temperatura de ignição. Assim, dados como possíveis incompatibilidades com outros compostos são de extrema importância para a prevenção de possíveis explosões ${ }^{38}$. Reich $^{39}$, utilizando DTA, avaliou a compatibilidade de formulações de explosivos obtidas a partir da mistura de polímeros com materiais altamente energéticos, avaliando a energia de ativação $\left(E_{a}\right)$ e a ordem de reação (n). Segundo o autor ${ }^{39}$, se a energia de ativação e a ordem de reação determinada para a decomposição térmica da formulação for igual à obtida pela análise individual do material energético, o sistema é considerado compatível.

\section{CONCLUSÃO}

As técnicas de análise térmica vêm sendo amplamente utilizadas no estudo da decomposição de materiais energéticos ${ }^{40}$. A maior vantagem em relação às outras técnicas deve-se à utilização de somente alguns miligramas da amostra. Estas técnicas podem ser usadas tanto na caracterização como no desenvolvimento de materiais energéticos, pois muitas informações sobre um explosivo podem ser obtidas antes de uma quantidade perigosa ser preparada.

Os parâmetros cinéticos obtidos por estas técnicas estão sendo utilizados para calcular a temperatura crítica de explosivos, para uso em modelagens térmicas com o objetivo de prever o tempo de explosão térmica em configurações reais e para avaliar a compatibilidade entre os componentes de uma formulação. A literatura mostra quais cuidados devem ser tomados, tanto na realização da análise quanto na comparação com outros dados.

Os resultados estão amplamente relacionados às condições experimentais e vários fatores, como tamanho e tipo da cápsula, e ainda o grau de confinamento, podem afetar o mecanismo da decomposição térmica da amostra. Diferentes mecanismos de decomposição térmica podem ser encontrados dependendo do contato dos produtos da decomposição com os produtos originais. Outras variáveis que podem mostrar influência nos resultados são as pressões internas; o tipo de atmosfera gasosa sob condição estática ou dinâmica; condições da amostra em relação à quantidade, granulometria, formato e disposição na cápsula.

O tipo de equipamento, os métodos de análise, as razões de aquecimento e a variedade de métodos de cálculo levam a uma grande diversidade de parâmetros cinéticos. A precisão da temperatura observada para o fenômeno em estudo é de extrema importância na medida cinética, portanto, a calibração do aparelho com referências que ofereçam respostas precisas tanto quanto possível é imperiosa.

Em meio às varias observações mencionadas anteriormente, verifica-se que a realização do estudo de decomposição térmica de um material inicia-se primeiramente pela escolha da técnica mais adequada para o trabalho. Etapas como a adoção dos parâmetros de trabalho, coleta de dados, escolha do modelo cinético para cálculo e interpretação dos resultados são decisivas para obtenção de um modelo mais preciso no estudo de um composto e, também, no desenvolvimento de novos materiais energéticos. Uma análise crítica deve ser realizada em relação aos parâmetros de trabalho mais adequados ao estudo em questão.

\section{AGRADECIMENTOS}

À CAPES pelo apoio financeiro e ao assessor pela forma criteriosa de avaliação e sugestões.

\section{REFERÊNCIAS}

1. Silva, G.; Pinheiro, G. F. P.; Iha, K.; Dutra, R. C. L.; Takahashi, M. F. K.; Reis, T. B.; Quim. Nova 2006, 29, 681.

2. Stankovic, M.; Filipovic, M.; Kapor, V.; J. Therm. Anal. Calorim. 1998, $52,439$.

3. Peng, D.; Chang, C. M.; Chiu, M.; J. Hazard. Mater. 2004, A114, 1; Zinn, J.; Rogers, R. N.; J. Phys. Chem. 1962, 66, 2646.

4. Mattos, C. E.; Moreira, E. D.; Dutra, R. C. L.; Diniz, M. F.; Ribeiro, A. P.; Iha, K.; Quim. Nova 2004, 27, 540; Liu, Z. R.; Yin, C. M.; Wu, C. H. Y.; Chang, M. N.; Propellants, Explos., Pyrotech. 2004, 11, 10.

5. Feng, H. T.; Mintz, K. J.; Aungsten, R. A.; Jones, D. E. G.; Thermochim. Acta 1998, 311, 105.

6. Lee, J. S.; Hsu, C. K.; Thermochim. Acta 2002, 392, 153; Rocco, J. A. F. F.; Lima, J. E. S.; Frutuoso, A. G.; Iha, K.; Ionashiro, M.; Matos, J. R.; Suárez-Iha, M. E. V.; J. Therm. Anal. Calorim. 2004, 75, 551.

7. Shehata, A. B.; Hassan, M. A.; Polym. Degrad. Stab. 2002, 77, 355

8. Koshigoe, L. G.; Shoemaker, R. L.; Taylor, R. E.; AIAA J. 1984, 22, 1600.

9. Kissinger, H. E.; J. Research of the National Bureau of Standards 1956, 57, 217; Coats, A. W.; Redfern, J. P.; Nature 1964, 201, 68; Rogers, R. N.; Morris, E. D.; Anal. Chem. 1966, 38, 412.

10. Rocco, J. A. F. F.; Lima, J. E. S.; Frutuoso, A. G.; Iha, K.; Ionashiro, M.; Matos, J. R.; Suárez-Iha, M. E. V.; J. Therm. Anal. Calorim. 2004, 77, 803.

11. Kishore, K.; Anal. Chem. 1978, 50, 1079.

12. Singh, G.; Felix, S.; Soni, P.; Thermochim. Acta 2005, 426, 131.

13. Long, G. T.; Vyazovkin, S.; Brems, B. A.; Wight, C. A.; J. Phys. Chem. B 2000, 104, 2570.

14. Bunyan, P.; Baker, C.; Turner, N.; Thermochim. Acta 2003, 401, 9.

15. Kissinger, H. E.; Anal. Chem. 1957, 29, 1702; Ozawa, T.; J. Therm. Anal. Calorim. 1970, 2, 301.

16. Kimura, J.; Kubota, N.; Propellants, Explos., Pyrotech. 1980, 5, 1.

17. Cropex, D. M.; Kemme, P. A.; Day, J. M.; Environ. Sci. Technol. 2002, $36,4346$.

18. Prabhakaran, K. V.; Bhide, N. M.; Kurian, E. M.; Thermochim. Acta 1995, 249, 249.

19. Kulkarni, P. B.; Purandare, G. N.; Nair, J. K.; Talawar, M. B.; Mukundan, T.; Asthana, S. N.; J. Hazard. Mater. 2005, A119, 53.

20. Mintz, K. J.; Jones, D. E. G.; Thermochim. Acta 1996, 284, 229; Ksiqzcazak, A.; Maranda, A.; Rosenkiewicz, D.; J. Therm. Anal. Calorim. 2000, 60, 97

21. Haberman, J.; Castorina, T. C.; Thermochim. Acta 1972, 5, 153.; Graybush, R. J.; May, F. G.; Forsyth, A. C.; Thermochim. Acta 1971, 2, 153.

22. Talawar, M. B.; Chhabra, J. S.; Agrawal, A. P.; Asthana, S. N.; Rao, K. U. B.; Singh, H.; J. Hazard. Mater. 2004, A113, 27.

23. Lukin, K. A.; Li, J.; Eaton, P. E.; Kanomata, N.; Hain, J.; Punzalan, E.; Gilardi, R.; J. Am. Chem. Soc. 1997, 119, 9591.

24. Singh, G.; Felix, S. P.; Soni, P.; Thermochim. Acta 2003, 399, 153.

25. Felix, S. P.; Singh, G.; Sikder, A. K.; Aggrawal, J. P.; Thermochim. Acta 2005, 426, 53 .

26. Blomouist, H. R.; US pat. Appl No 2031131998

27. Östmark, H.; Bergman, H.; Ekvall, K.; Langlet, A.; Thermochim. Acta 1995, 260, 201. 
28. Singh, G.; Felix, S. P.; Pandey, D. K.; Thermochim. Acta 2004, 411, 61.

29. Östmark, H.; Bemm, U.; Bergman, H.; Langlet, A.; Thermochim. Acta 2002, 384, 253.

30. Estes, E. D.; Estes, W. E.; Hatfield, W. E.; Hodgson, D. J.; Inorg. Chem. 1975, 14, 106

31. Talawar, M. B.; Sivabalan, R.; Senthilkumar, N.; Gurumallesh, P.; Asthana, S. N.; J. Hazard. Mater. 2004, A113, 11.

32. Sikder, A. K.; Sikder, N.; J. Hazard. Mater. 2004, A112, 1.

33. Simpson, R. L.; Urtiew, P. A.; Ornellas, D. L.; Moody, G. L.; Scribner, K. J.; Hoffman, D. M.; Propellants, Explos., Pyrotech. 1997, 22, 249; Bottaro, J. C.; Chem. Ind. 1996, 1, 249; Bunyan, P.; Baker, C.; Turner, N.; Thermochim. Acta 2003, 401, 9.

34. Oxley, J.; Smith, J.; Zheng, W.; Rogers, E.; Coburn, M.; J. Phys. Chem. 1997, A101, 4375.
35. Rongzu, H.; Zhengquan, Y.; Yanjun, L.; Thermochim. Acta 1988, 123, 135; Yi, X.; Rongzu, H.; Chaoqing, Y.; Guofu, F.; Jihua, Z.; Propellants, Explos., Pyrotech. 1992, 17, 298.

36. Tonglay, Z.; Rongzu, H.; Yi, X.; Fuping, L.; Thermochim. Acta 1994, 244, 171

37. Semenov, N. N.; Some Problems of Chemical Kinetics and Reactivy, $2^{\text {nd }}$ ed., Princeton University Press: Princeton, 1958.

38. Zinn, J.; Mader, C. L.; J. Appl. Phys. 1960, 31, 323; Hussain, G.; Rees, G. J.; Fuel 1995, 74, 273.

39. Reich, L.; Thermochim. Acta 1973, 5, 433.

40. Silva, G.; Mattos, E. C.; Nakamura, N. M.; Iha, K.; Quim. Nova 2004, 27, 889 